Proceedings of the 2011 Winter Simulation Conference

S. Jain, R.R. Creasey, J. Himmelspach, K.P. White, and M. Fu, eds.

\title{
REAL TIME PERFORMANCE MEASUREMENT FOR BATCH CHEMICAL PLANTS
}

\author{
Pradeep Suresh \\ The Dow Chemical Company \\ B1603, 2301 N. Brazosport Blvd. \\ Freeport, TX 77541 USA
}

\author{
John M. Wassick \\ Jeff Ferrio \\ The Dow Chemical Company \\ 1776, Michigan Operations \\ Midland, MI 48640, USA
}

\begin{abstract}
The objective of this work was to develop and demonstrate batch process optimization tools that can be deployed for use in a manufacturing environment. The work specifically addresses the lack of tangible real time performance measures for batch process operations in literature and industry. Such performance measures need to account for real time adherence to production schedule, capture the impact of unexpected events and measure the consequence of such performance on meeting product orders or desired inventory levels. This work combines real time plant data and the concept of an 'Online Simulation' to continuously estimate probable end states proceeding from the current time. Such a real time performance measure successfully captures deviation from expected performance and its impact on process deliverables. This aids real time decision making and process improvements for meeting productivity targets and maximizing economic value.
\end{abstract}

\section{MOTIVATION}

The term 'performance measure' could refer to many different things for a batch process. In this work, the subject of investigation was a measure of performance for the entire process or plant rather than a single unit operation in isolation or a single batch. Performance was not measured for example, by how tightly controlled the temperature of a reactor was, or if the duration of a loading operation was unexpectedly long. Instead, this work was motivated by the need for a more tangible real time performance measure that correlated the plant performance to its deliverables (e.g. customer order fulfillment, desired product inventory level). Existing literature and commercial product offerings from various vendors were surveyed for ideas.

Commercial software products that fall under the category of Manufacturing Execution Systems (MES) typically are dashboard type applications that allow monitoring real time operations. These products display operating status of various equipments, inventories, personnel, etc. This can be very useful to track performance of the monitored entities in real time. Siemens IT XHQ (Siemens AG 2010) imports processing times, batch quantity, asset utilization for each equipment, material and control step. It compares these parameters with given targets and reports deviations, gaps and potential root causes. ABB 800xA Batch Management (ABB 2011) is a recipe and control management framework for designing and monitoring batch processes. It has capabilities that enable dynamic optimization of recipe parameters as well. It also maintains electronic batch records of production campaigns. Emerson Syncade Management Information Portal (Emerson Process Management 2011) contains a document, recipe and process control analytics display with customizable views and has a lot of similar capabilities as the ABB MES. Honeywell's Plant Performance Monitor (Honeywell Process Solutions 2011) comprises a set of functional modules designed to determine the efficiency of a particular piece or stage of equipment as it evaluates the overall unit efficiency. Most of these MES applications also report Key Performance Indicators (KPI) 
such as Overall Equipment Effectiveness (OEE), Cycle time, etc. The indicators of performance used in industrial applications are consistent with those found in the literature in areas of dynamic optimization, manufacturing research (Eurobios and SCA Packaging 2005; Ahmad et al. 2005) and process simulation. These measures, though useful, do not help predict the impact of process deviations on process performance.

\section{PROPOSED SOLUTION}

When a process experiences unexpected events, quite often, product order delivery dates are missed or minimum inventory levels are violated. Such consequences may result in penalties and losses if not detected and corrected for at the earliest. It would in fact be quite useful to have a performance measure that can measure the impact of the process performance deviations in tangible terms such as order status or resulting inventory level deviations. This work proposes such a measure to fulfill this unmet need in batch plant performance measurement. Figure 1 shows the schematic of the proposed solution strategy.

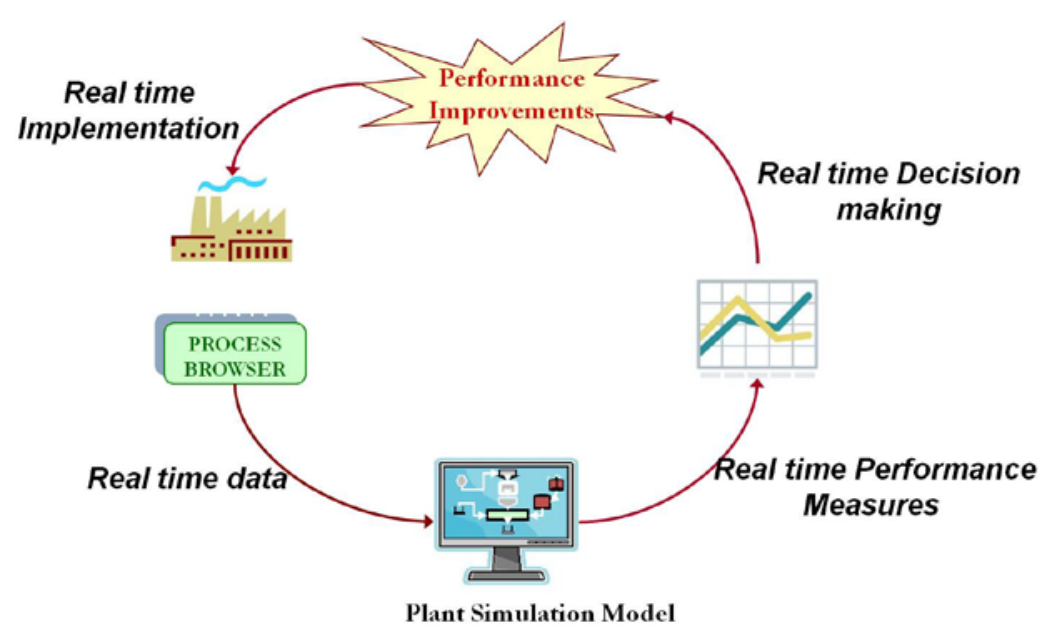

Figure 1: Solution strategy for performance measurement of batch plant

In the proposed approach, plant data is fed in real time to a plant simulation model through a process browser and an interface. The simulation models used in this work were Discrete Event Simulation (DES) models of the processes developed using ExtendSim (Diamond et al. 2010). The simulation model incorporates the various uncertainties associated with the plant operations and mimics plant operation in terms of material inventories, processing times of operations and the various activities involved in manufacturing the different products made at the plant. Real time plant data is used to initialize the plant simulation model and the remaining production campaign is simulated. Results of the simulation such as batch completion times for each batch in the product campaign, final inventory levels, unexpected bottlenecks, wait times and resource conflicts are reported and displayed through an interface. Additionally, since nondeterministic processing times are incorporated in the DES, multiple Monte-Carlo simulation runs are performed to determine the probability of filling product orders at each time point. These tangible metrics of process performance could now trigger real time initiatives to improve performance if necessary. They could also trigger a reactive rescheduling scheme to shuffle production orders. It might even help in renegotiating contracts and delivery times with customers ahead of time with a reasonable confidence level. This interactive mechanism could be run at a user desired frequency.

The proposed approach was compared with existing literature in the areas of production scheduling under uncertainties and dynamic (or reactive) rescheduling. Perhaps the work closest in philosophy to the current work is that involving FOSS (Framework for Online Scheduling and control System) (Ishii and 
Muraki 1997). This work extended previous work in this area that had given rise to PVSS (Process Variability based Scheduling System) (Ishii and Muraki 1996). PVSS was an online rescheduling algorithm that was triggered by processing time variations in tasks and operations. It did not handle uncertainties other than processing time variations, for example equipment breakdown, etc. FOSS, on the other hand, was a comprehensive online rescheduling framework (FOSS) that could handle most types of uncertainties in a batch plant. It had process monitoring modules that sensed changes in online process data as well as other modules that monitored equipment, order status etc. To alleviate the problem of triggering the rescheduling algorithm for minor changes sensed by the monitoring modules, process changes were input to a discrete event simulation of the process. The simulation was deterministic in nature and simulated the effects of process deviations on scheduled events without accounting for process variability.

DES of plants has also been used to analyze product schedules at the start of product manufacture or to evaluate proposed schedule changes when processes experience disruptions (Honkomp, Mockus, and Reklaitis 1999; Chong, Sivakumar, and Gay 2003). Another philosophy towards estimating the consequences of process performance deviations is from historical data (Kim, Jung, and Lee 1996; Kim and Lee 1997). If the sources of process disruptions can be catalogued along with their frequency and impact on process deliverables, this could be a viable approach, albeit sometimes tedious. Such a data driven method could also give rise to artificial cause-effect correlations that do not actually exist in the process.

\section{ONLINE SIMULATION OF PLANT PERFORMANCE}

The concepts described in the proposed approach for real time performance measurement were demonstrated at a chemical plant of The Dow Chemical Company. A high level overview of the process operations is described here. The plant had four production trains with three main processes. Raw materials (RM1 and RM2) are loaded from raw material stations during Process 1, followed by transfer to Process 2 and subsequently to Process 3. Pre-Adds (PA) are added during Process 3 prior to the material transfer from the Process 2 and the batch is processed. Product stream from Process 3 is packaged for storage or delivery. Four different types of PAs can be made in Process 4 and stored for use by the four production trains. Different products made in each train use varying quantities of RM1, RM2 and one of the PreAdds based on their recipe. The four trains share RM1 and RM2 conveying, RM3 production and storage, small adds (other raw materials in trace quantities) and packaging systems. The four trains and the PA production process (Process 4) are operated based on production schedules to meet demand in the form of product orders. Figure 2 shows the schematic of operations in this batch plant. The following section describes the discrete event simulation model of this process built using ExtendSim along with the associated assumptions and simplifications.

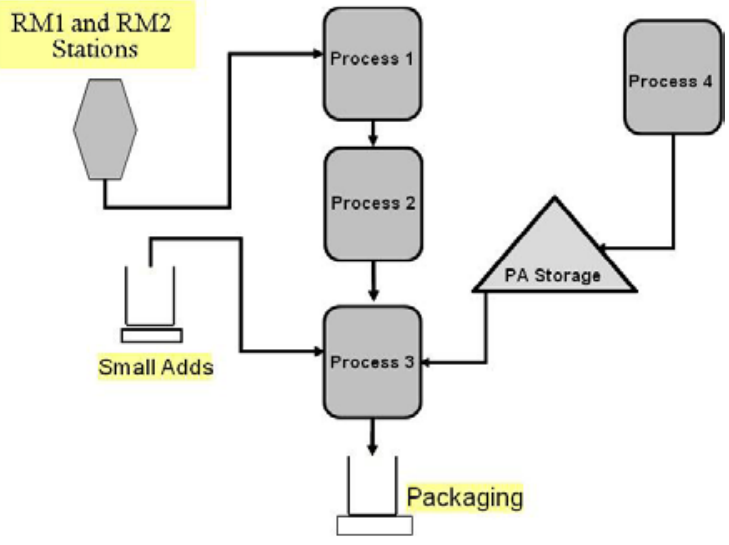

Figure 2: Schematic of Batch Production process 


\subsection{Simulation model}

The ExtendSim model of the generic batch plant described in the previous section was built. A basic block diagram used for modeling the processes involved in the simulation is shown in Figure 3.

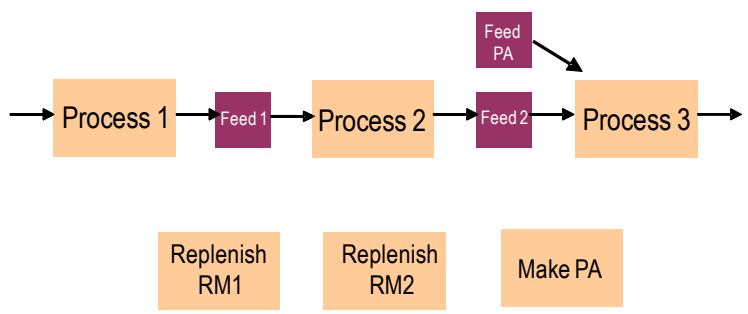

Figure 3: Simulation model logic

While the top line of processes in Figure 3 represent the processes in each train (and hence was defined four times, one for each train), the bottom line of processes are those common to all the trains. During a feed step, both the upstream and downstream equipment are busy with material transfer occurring between them. They are not available for other operations or batches during this 'handshake' step. The raw material replenishment processes are shared between the trains and inventory driven having fixed setpoints. It can be seen that the processes involved are for the most part, linear in nature, i.e. process units are mostly connected in series. Therefore the overall cycle time should have been approximately equal to the sum of the individual processing times involved. However, shared resources such as the raw material stations and the PA storage tanks make this model nonlinear. The processing times of the various processes depend on the product and duration of the various manual tasks involved in these processes. This induces appreciable variability in the processing times involved and hence cycle times. Additionally, unexpected events, changes in production schedules, and product transitions contribute further to the uncertainty and variability associated to predicting batch completion times.

The time distributions for the various processes were regressed from actual historical data viewed through IP.21 (Aspen IP.21 Process Browser 2011), Batch.21 (Aspen Production Record Manager 2011) and fitted using JMP (SAS Institute 2011). Inventory levels of the different raw materials and products were initialized (from values in the plant at a particular point in time) in the simulation along with their maximum and minimum levels. The equipments list in the process as well as the production schedules for PA production and the four production trains were also initialized. A sample list of product orders with expected delivery dates and product amounts was also used as input to the simulation model (Table 1).

Table 1: Order information for the simulation model

\begin{tabular}{|c|c|c|}
\hline Delivery Date & Product ID & Amount (lbs) \\
\hline 1/12/2010 0:00 & 1 & 15000 \\
\hline 1/14/2010 6:00 & 2 & 70000 \\
\hline 1/14/2010 6:00 & 3 & 80000 \\
\hline 1/15/2010 0:00 & 4 & 115000 \\
\hline
\end{tabular}

The process recipes for the different products made at this plant was also captured in the simulation model. The impact of manual tasks, shared equipment/inventory conflicts and all other sources of uncertainty were captured in terms of the process time distribution data collected from the plant. At the end of a simulation run, the model reported batch completion times by train, average batch completion time and 95\% confidence interval on the batch completion times. The last two output variables are applicable when variability in batch times was modeled. Other outputs of the simulation were the probability of filling customer orders calculated based on the current date and inventory levels of required product on the 
delivery date and time. If the simulated inventory level of the required product was enough to fill the order, the simulation run was considered successful towards filling the order and if not, counted as unsuccessful. On simulating enough Monte Carlo replicates, the probability of filling each order was reported. This estimated probability captures the consequence of the variability and uncertainty in batch plants.

Another capability added to the simulation logic was to start the simulation from a non-empty state or to have batches (in different trains) in progress at the start of the simulation. The details of the Work in progress (WIP) batches were captured in an input data table shown in Table 2. The 'WIP state' in this table is a binary variable with a value of 1 indicating the presence of a batch in progress in that process. The 'Time Done' variable in this table captures the duration since the start of this process for this batch. 'Product ID' is the identifier for the product the batch in progress is making. The simulation logic adjusts the processing time left and batch size of the WIP processes based on this table.

Table 2: Work in Progress (WIP) state input to the simulation model

\begin{tabular}{|c|c|c|c|}
\hline Process & WIP state & Time Done & Product ID \\
\hline Process 1 (Train 1) & 0 & 0 & \\
\hline Feed 1 (Train 1) & 0 & 0 & \\
\hline Process 2 (Train 1) & 0 & 0 & \\
\hline Feed PA (Train 1) & 0 & 0 & \\
\hline Feed 2(Train 1) & 0 & 0 & \\
\hline Process 3 (Train 1) & 0 & 0 & \\
\hline$\ldots \ldots \ldots$ & $\ldots \ldots$ & $\ldots \ldots$ & $\ldots \ldots \ldots$ \\
\hline Process 3 (Train 4) & 0 & 0 & \\
\hline Make PA & 0 & 0 & \\
\hline Replenish RM1 & 0 & 0 & \\
\hline Replenish RM2 & 0 & 0 & \\
\hline
\end{tabular}

This simulation model could be run standalone to simulate production schedules, investigate waiting times and bottlenecks or to judge probabilities of fulfilling product orders prior to start of production based on initial inventory and equipment levels. Figure 4 shows the simulated and the actual completion times of the batches from a historical start date.

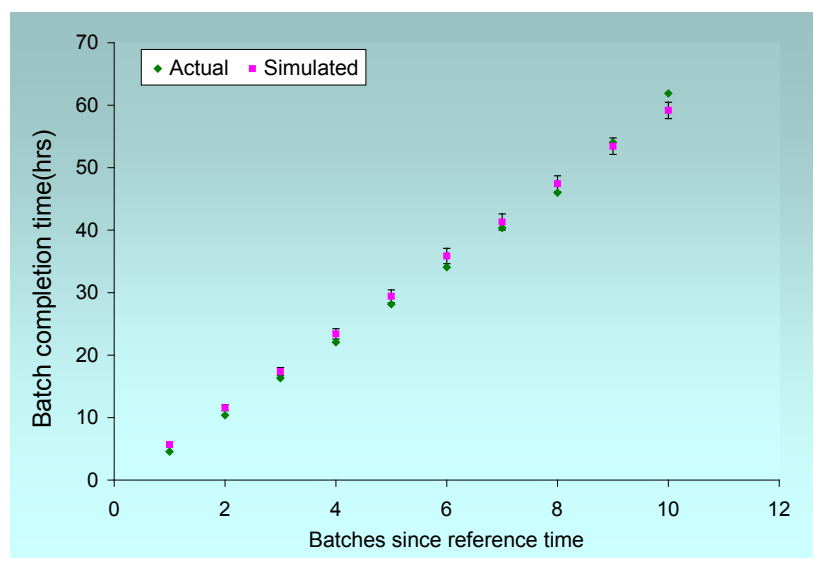

Figure 4: Simulated and actual batch completion times

The simulated and actual waiting times for downstream operations or 'blocking' duration were also similarly validated. The accuracy of the simulation model in representing the actual process operations were thus verified and validated before being put to use for performance measurement. 


\subsection{Integrating Real Time Plant data and Simulation model}

The simulation model described in the previous section imports data from text files to initialize inventory level, WIP states, order and schedule information. These text files could potentially be automatically generated from real time data from the plant using IP.21 and a VBA interface. Though this connection was not completely established as part of this work, the basic procedural building blocks were setup. The code to create a WIP state table from the IP.21 data, given an input date, was developed. This code could be extended to automatically retrieve the various inventory levels from the IP.21 data, remaining schedule of batches and product order data from ERP software products such as SAP. For the current case study, this input information was manually created as text files for input to the DES.

\section{RESULTS}

Figure 5 shows the output of the proposed strategy at a particular time point in production. These probability numbers were generated based on multiple Monte Carlo replications of the plant simulation model with the plant state at that time point as the input. The plot is updated in user defined time intervals and acts a tangible measure of current plant performance. At the same time, it helps support strategic decision making that might be necessary during production to meet customer demand and production targets. It is envisioned that this would also feedback into and trigger a dynamic rescheduling framework that can make necessary production schedule changes to meet customer product orders. Additionally, the proposed strategy estimates final inventory levels of raw materials as well as products (Figure 6). These estimates can be tracked over time to forecast unexpected raw material demand or to trigger schedule optimization in 'make to stock' strategies.

The other capabilities of the proposed strategy will be presented here in the form of different scenarios that could occur at the plant and how the proposed solution measures the impact of these scenarios. In these scenarios, deterministic processing times were used in the DES to simulate particular situations and the system's response to them. For the purpose of the case study, Train 1 is making Product 1 and Train 2 is making Product 2. We will further assume that Product 1 requires PA1 and PA2, Product 2 requires PA2 and PA3 for their manufacture.

\begin{tabular}{|c|c|c|c|c|c|}
\hline \multicolumn{4}{|c|}{ Real Time Order Status } & \multicolumn{2}{|c|}{ Current Date: 6/29/2011 9:03:15 } \\
\hline Order No. & Product & Quantity & $\begin{array}{l}\text { Delivery } \\
\text { Date }\end{array}$ & $\begin{array}{l}\text { Order Filled } \\
\text { (Y/N) }\end{array}$ & $\begin{array}{l}\text { Probability of } \\
\text { Filling Order on Time }\end{array}$ \\
\hline 2442622 & 2 & $20,000.00$ & $6 / 28 / 2011$ & $\mathbf{Y}$ & \\
\hline 2442623 & 4 & $41,250.00$ & $6 / 29 / 2011$ & $\mathbf{Y}$ & \\
\hline 2442651 & 3 & $10,000.00$ & 7/1/2011 & $\mathbf{N}$ & $60 \%$ \\
\hline 2442600 & 3 & $35,500.00$ & $7 / 2 / 2011$ & $\mathrm{~N}$ & $86.67 \%$ \\
\hline
\end{tabular}

Figure 5: Snapshot of Order fulfillment status output 


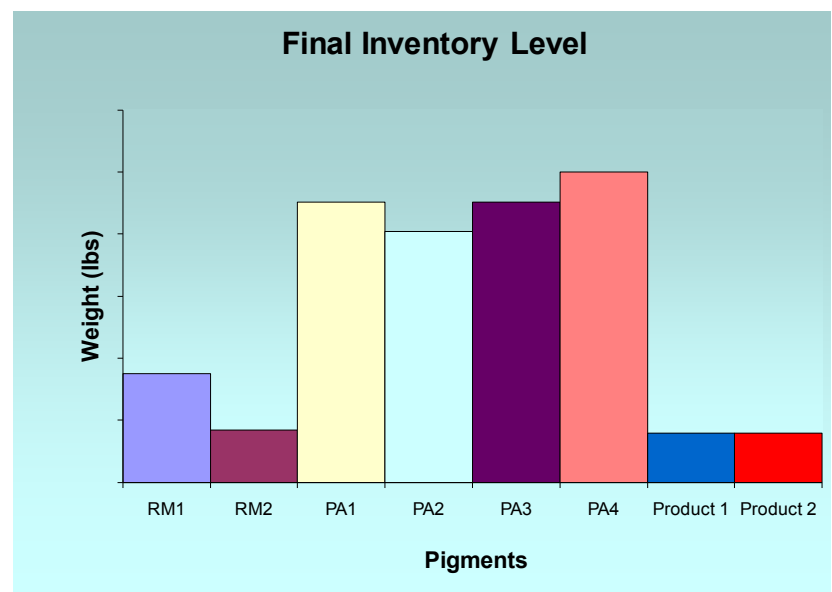

Figure 6: Estimate of final inventories at an instant of time

\subsection{Scenario 1}

Figure 7 shows a Gantt chart of a batch in Trains 1 and 2. Per the original production schedule, there were no equipment or inventory conflicts expected between the two trains. In this scenario, a delay in Process 1 on Train 1 is emulated. This delay results in a resource conflict between two trains demanding the same PA (PA2) at the same time. We assume that there is enough PA2 in the inventory and the conflict is for the feeding PA2 into Process 3. With traditional performance measurement dashboards and techniques, this situation would not be detected until it actually occurs. At this point, it is too late to take any corrective action and one of the trains ends up waiting for PA2 (depending on which train gets to the feed step first).

With the proposed performance measurement technique, this situation can be foreseen as early as the 3 hour mark (depending on how frequently the simulation model is run). Since this scenario uses deterministic processing times, by simulating forward in time at the end of the load step in Train 1, it can be predicted with certainty that a potential conflict would occur in the Feed PA step. In a real world equivalent of this scenario, a probability distribution of the delay could be reported at time instances when the simulation is used to look ahead. When the probability of a non-zero delay is higher than a threshold value (depending on the risk tolerance of the user), corrective action of some form can be triggered. For proof
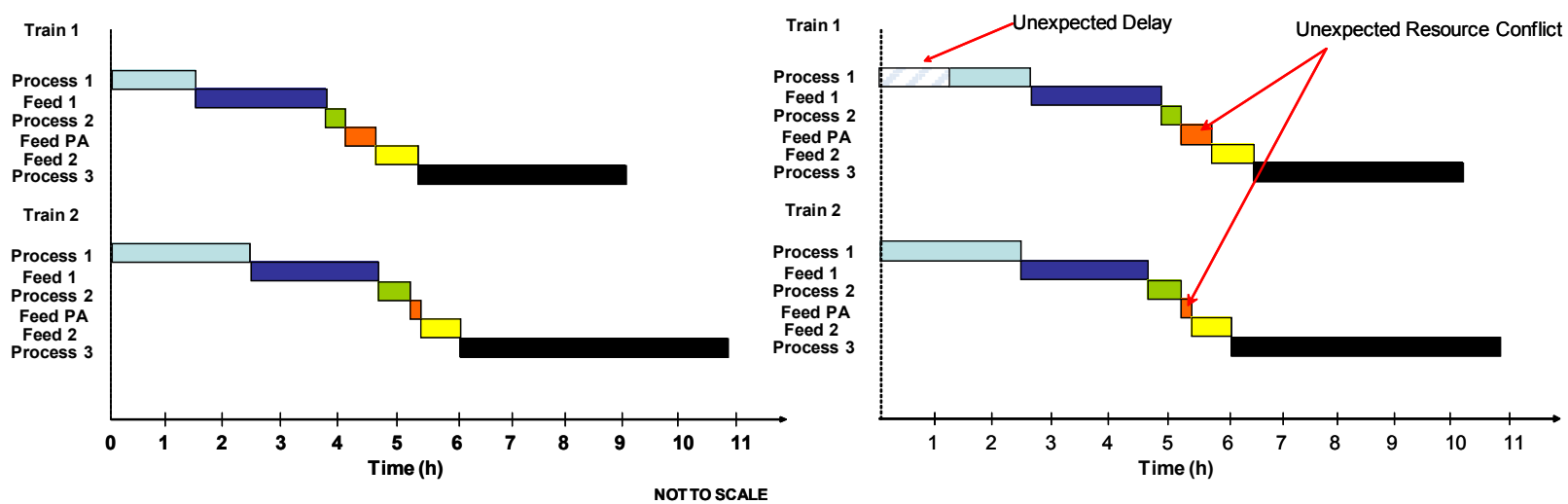

Figure 7: Expected (left) and actual (right) outcomes in Scenario 1 
of concept purposes, input files such as WIP state, inventory level, remaining schedule of batches etc were manually created based on the Gantt chart at time instants $t=0,1,2,3$ hours. The VBA interface was automated to send these input files periodically the DES, execute the DES and capture the results back in an Excel interface. It can be seen from Figure 8 that the estimated wait time for PA from the proposed approach is non zero at $t=3$ hours (batch is off schedule). If the performance measurement tool were run more aggressively at shorter time intervals, one could have observed this non zero wait much earlier. As mentioned earlier, the wait time could be pointed out with certainty due to the deterministic processing times in the scenario.

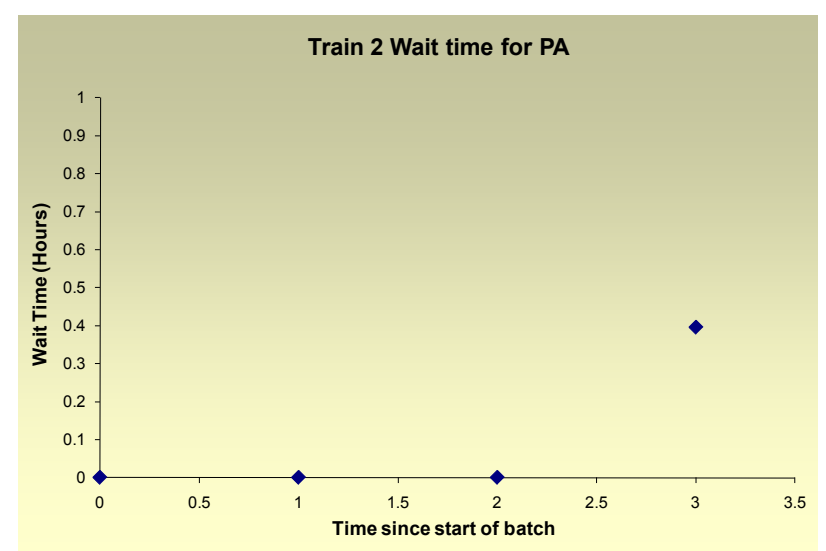

Figure 8: Estimated wait time for PA for Train 2 in Scenario 1

Figure 9 shows the estimated deviation from expected batch completion times for the batch in Train 1 and Train 2. As described earlier, the estimate at $t=1$ for both trains assumes that Process 1 in Train 1 still can complete at the scheduled end time. At $t=2$ hours, the estimated completion time for the batch in Train 1 is pushed back (resulting in a large positive deviation, implying batch is off schedule), but that in Train 2 is on schedule. Only at $t=3$ hours, is the full magnitude of the delay in Process 1 realized, since now, a potential PA conflict between the trains pushes the batch completion time in Train 2. The impact of the delay in this scenario isn't very significant. One can easily envision significant conflicts and delays being predicted a priori by this performance measurement technique.
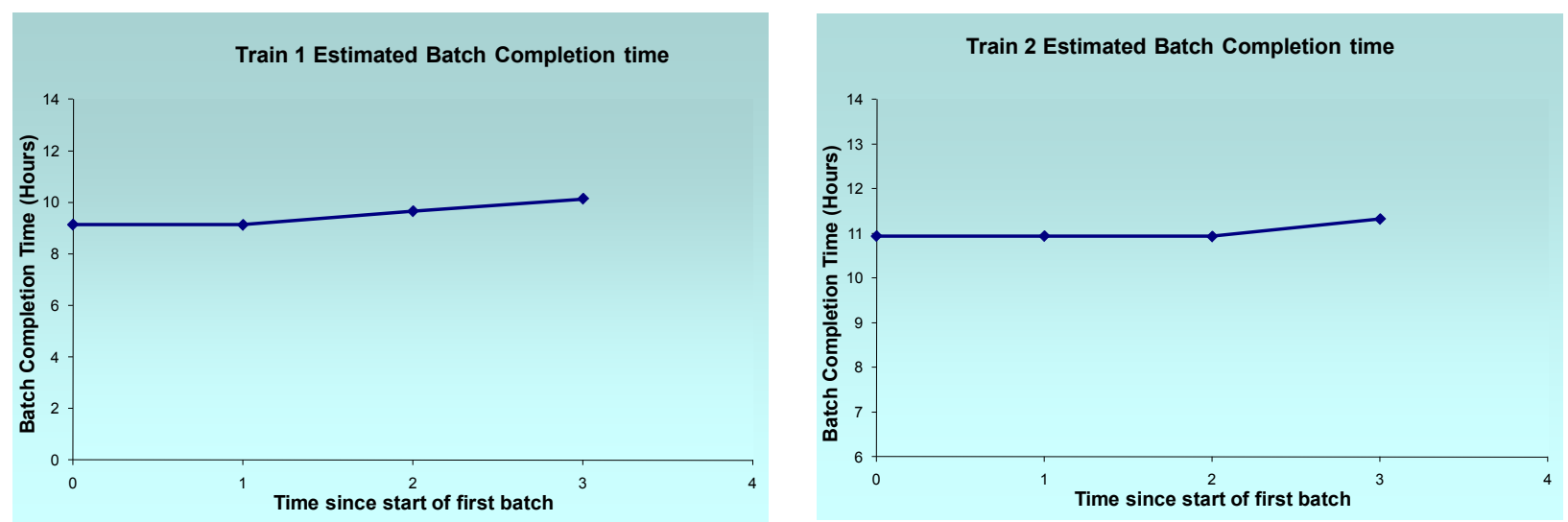

Figure 9: Batch completion times for Train 1 (left) and Train 2 (right) in Scenario 1 


\subsection{Scenario 2}

Scenario 2 emulates a situation where a delay in the Process 1 in Train 1 blocks Process 4 (Make PA) from finishing and transferring to storage. The inventory levels of PA1 are such that two batches in Train 1 need to consume PA1 so that the batch of PA1 produced in Process 4 can be emptied into the storage tank. If the loading step in Train 1 is delayed long enough that the second batch in Train 1 does not finish by the time the Process 4 produces a batch of PA1, the Process 4 schedule experiences an unexpected delay. With the proposed performance measurement technique, this conflict can be detected and corrective action can be implemented to avoid it. This scenario is shown in Figure 10.

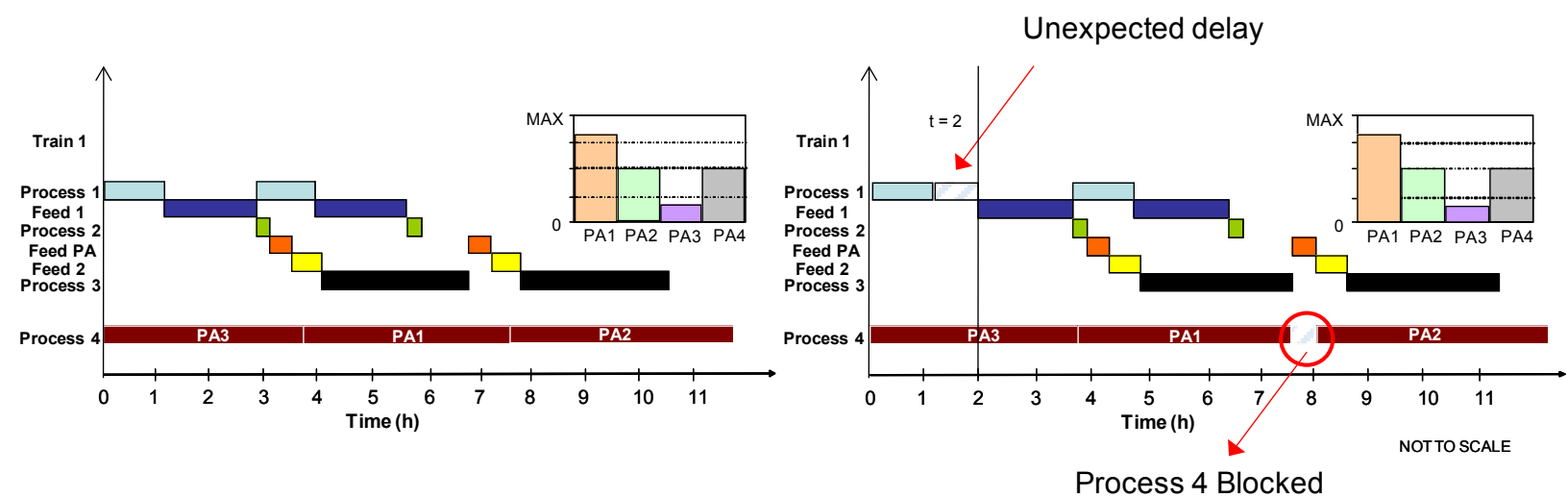

Figure 10: Expected (left) and actual (right) outcomes in Scenario 2

Input files were manually created to denote WIP states, inventory levels etc for each time $=0,1$ and 2 hours. The simulation model was run with each of these sets of input files to generate output files. Figure 11 and Figure 12 show the output from the proposed performance measurement approach, which is the expected blocking times and the expected batch completion times.

One can similarly envision a scenario where an unexpected delay in the Process 4 schedule could lead to one of the trains waiting (or being starved) for Process 3 to start. This might only happen when the PA inventory levels are insufficient for Process 3 to use.

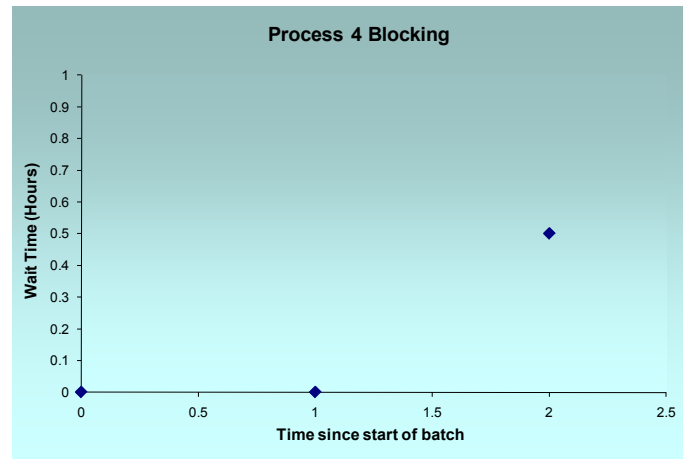

Figure 11: Process 4 blocking in Scenario 2 


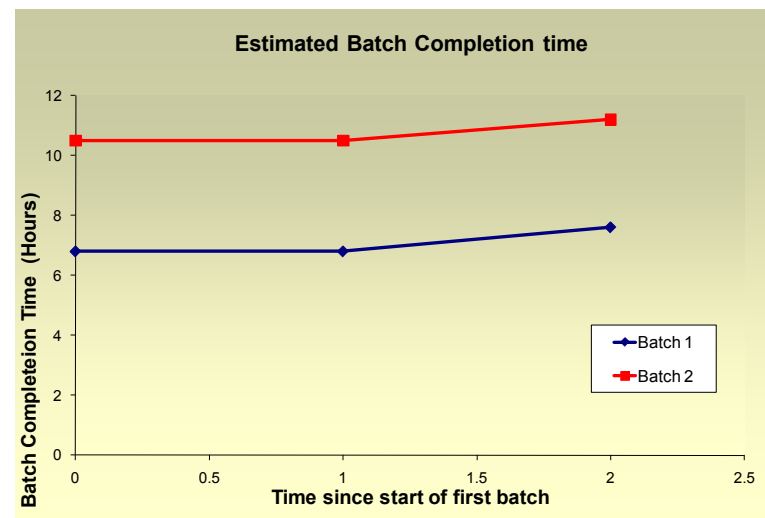

Figure 12: Batch completion times for Train 1

\section{SUMMARY}

This work has proposed a mechanism for real time performance measurement of batch chemical plants. The mechanism comprises of using real time plant data to initialize a plant simulation model and estimating the end state of a batch campaign. This provides a real time measure of batch completion times, probability of meeting customer demand, final inventory levels and potential resource conflicts. These measures can then be used to propose corrective action such as dynamic rescheduling of batches and aid decision making in real time to reach production targets and an economic optimum. The solution strategy is intuitive and can be completely automated providing tangible, practically useful information to a plant engineer. The main caveat of the approach is that the simulation model has to be representative of the production environment at the plant. The DES used as the dynamic plant model in the proposed solution is fully capable of accounting for real world complexities and uncertainties that surround production environments. This makes the proposed real time performance measurement solution a valuable time and cost saving asset for batch chemical plants.

\section{REFERENCES}

ABB, Process Automation Division, Vasteras, Sweden. 2011. Accessed May 31. http://www.abb.com/product/seitp334/f468ed41209d120a8525719b00622a83.aspx.

Ahmad, M., N. Dhafr, R. Benson and B. Burgess. 2005. "Model for establishing theoretical targets at the shop floor level in specialty chemicals manufacturing organizations." Robotics and ComputerIntegrated Manufacturing 21:391-400

Aspen IP.21 Process Browser, Aspen Technology Inc., Massachusetts, USA. 2011. Accessed May 31. http://www.aspentech.com/products/aspen-web21.cfm.

Aspen Production Record Manager (formerly Aspen Batch.21), Aspen Technology Inc., Massachusetts, USA. 2011. Accessed May 31. http://www.aspentech.com/products/aspen-batch21.cfm.

Chong, C., A. Sivakumar and R. Gay. 2003. "Simulation based scheduling for dynamic discrete manufacturing." In Proceedings of the 2003 Winter Simulation Conference, edited by S. Chick, P. Sánchez, D. Ferrin, and D. Morrice, 1433-1439. Piscataway, New Jersey: Institute of Electrical and Electronics Engineers, Inc.

Diamond, B., P. Diamond, S. Lamperti, D. Krahl, C. Sackett, A. Nastasi, C. Damiron, P. Tag and K. Hansen. 2010. ExtendSim User Guide V8. Imagine That Inc. Accessed May 31. http://www.extendsim.com.

Emerson Process Management, Missouri, USA. 2011. Accessed May 31. http://www2.emersonprocess.com/en-US/brands/syncade/Pages/Syncade.aspx. 
Eurobios and SCA Packaging, "Agent-Based Factory Modeling, a Case Study". 2011. Accessed May 31. http://www.agentlink.org/resources/webCS/AL3_CS_002_EUROBIOS.pdf.

Honeywell Process Solutions, Phoenix, AZ, USA. 2011. Accessed May 31. http:/hpsweb.honeywell.com/Cultures/enUS/Products/ControlApplications/AdvancedSolutionsPower Generation/AdvancedEnergySolutions/default.htm.

Honkomp, S. J., L. Mockus and G. V. Reklaitis. 1999. "A framework for schedule evaluation with processing uncertainty", Computers and Chemical Engineering 23:595-609

Ishii, N. and M. Muraki. 1997. "A generic framework for an on-line scheduling and control system in batch process management", Computers and Chemical Engineering 21 (11): 1291-1310.

Ishii, N. and M. Muraki. 1996. "A Process Variability based online scheduling system in multiproduct batch process", Computers and Chemical Engineering 20 (2):217-234.

Kim, M., J. H. Jung and I. Lee. 1996. "Intelligent scheduling and monitoring for multi-product networked batch processes", Computers and Chemical Engineering 20: S 1149-S 1154.

Kim, M. and I. Lee. 1997. "Rule-Based Reactive Rescheduling System for Multi-Purpose Batch Processes," Computers and Chemical Engineering 21:S1197-S1202.

SAS Institute Inc. 2011. JMP®. Accessed May 31. http://www.jmp.com.

Siemens AG, Industry Automation, Germany. 2011. Accessed May 31. http://www.energy.siemens.com/fi/en/industries-utilities/oil-gas/products-systems-solutions/xhqoperations-intelligence.htm.

\section{AUTHOR BIOGRAPHIES}

PRADEEP SURESH is a Senior Engineer at The Dow Chemical Company. He received his Bachelors in Chemical Engineering from Indian Institute of Technology (IIT)-Madras, India and a Ph.D. in Chemical Engineering from Purdue University. His research interests are in application of concepts in operations research, artificial intelligence and intelligent systems to solve industrial scale problems. He can be reached atpsureshbabu@dow.com.

JOHN M. WASSICK is a Fellow in the Core R\&D division of The Dow Chemical Company. He holds a Ph.D. in Electrical Engineering from Michigan State University. His current research interests include large scale optimization, optimization under uncertainty, advanced process automation and control, supply chain modeling and optimization, and financial engineering. He can be reached through e-mail at jwassick@dow.com.

JEFF FERRIO is a Research Scientist in the Core R\&D division of The Dow Chemical Company. He holds a Ph. D. in Chemical Engineering from Michigan Technological University. His research interests include process optimization, scheduling optimization, process control, modeling and simulation. He can be reached atjFerrio@dow.com. 